\title{
Generation and amplification of pulsed Bessel beams by seeding an optical parametric amplifier
}

\author{
M. Clerici, ${ }^{1,4}$ O. Jedrkiewicz, ${ }^{1,4, *}$ E. Rubino, ${ }^{1,4}$ D. Faccio, ${ }^{1,4}$ L. Tartara, ${ }^{2,4}$ V. Degiorgio, ${ }^{2,4}$ and \\ P. Di Trapani ${ }^{1,3,4}$ \\ ${ }^{1}$ Consorzio Nazionale Interuniversitario per le Scienze fisiche della Materia (CNISM) and Department of Physics and \\ Mathematics, Università dell'Insubria, Via Valleggio 11, IT-22100 Como, Italy \\ ${ }^{2}$ Consorzio Nazionale Interuniversitario per le Scienze fisiche della Materia (CNISM) and Department of Electronics, \\ Università degli Studi di Pavia, Via Ferrata 1, IT-27100 Pavia, Italy \\ ${ }^{3}$ Department of Quantum Electronics, Vilnius University, Sauletekio Avenue 9, Building 3, LT-10222 Vilnius, \\ Lithuania \\ ${ }^{4}$ Virtual Institute for Nonlinear Optics, Centro di Cultura Scientifica Alessandro Volta, Villa Olmo, Via Simone \\ Cantoni 1, IT-22100 Como, Italy \\ *Corresponding author: ottavia.jedrkiewicz@uninsubria.it
}

\begin{abstract}
By using two very different seed pulses we demonstrate that the spatiotemporal gain properties of a $\chi^{(2)}$ optical parametric amplifier can be exploited as an efficient conical reshaping mechanism leading to the generation and amplification of a pulsed Bessel beam.
\end{abstract}

Conical waves such as Bessel X waves (BXW) [1] and pulsed Bessel beams (PBBs) [2] are the polychromatic pulsed generalization of the monochromatic Bessel beam. They have recently attracted considerable interest for their potential applications, ranging from optical communications, metrology, and spectroscopy (see [3] and references therein) to extreme nonlinear optics [4]. Although they appear spontaneously in many nonlinear interactions [5-8], or can be created by linear optical methods [9], the ability to efficiently generate a wide family of conical waves has been untill now far from being achieved.

It has been theoretically shown that the optical parametric amplifier (OPA) gain, which is dictated by the energy and momentum conservation laws, i.e., the phase-matching conditions [10], can support the amplification of conical wave packets thanks to the angular dispersion of the phase-matched radiation [11-13]. Moreover, vacuum seeded OPA generates radiation with intriguing conical spatiotemporal coherence properties [14], which can be exploited for conical pulse generation via a self-locking mechanism [15].

In this Letter, we experimentally demonstrate the possibility of efficiently generating and amplifying particular conical wave packets, i.e., PBBs, thanks to the pulse reshaping mechanism that occurs in a coherently seeded OPA. The PBB is a conical pulse having all the temporal frequencies distributed along a constant transverse wave vector component, i.e., with the amplitude front perpendicular to the propagation direction [16].

We start by describing a simple graphical representation of the spatiotemporal spectral characteristics of the radiation generated in parametric amplification processes. This can be obtained considering the spectral properties of the perfectly phase-matched radiation in the $\left(k_{\perp}, \omega\right)$ domain, where $k_{\perp}$ and $\omega$ are, respectively, the wave vector components orthogonal to the pump propagation direction and the frequency of the amplified radiation. Phase-matching curves for our experimental parameters, i.e., for a type I beta barium borate (BBO) crystal pumped by a $405 \mathrm{~nm}$ carrier wavelength pump pulse, are shown in Fig. 1(a). Different curves correspond to different crystal angular tuning conditions, i.e., for internal angles between the pump wave vector and crystal optical axis $\left(\theta_{t}\right)$ ranging from $27^{\circ}$ to $39^{\circ}$.

Each phase-matching curve, or section of it, if coherently excited, results in a conical pulse. For in-
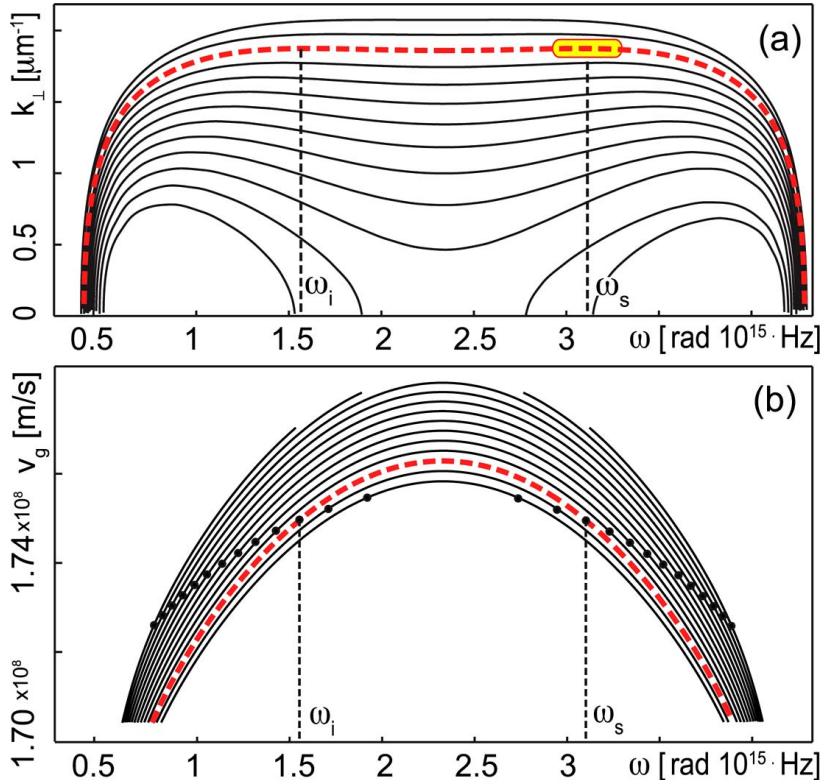

Fig. 1. (Color online) (a) Phase-matching curves for a type I BBO crystal pumped by a $405 \mathrm{~nm}$ pulse. The dashed curve corresponds to the experimental conditions and the circled section indicates the experimentally generated PBB; (b) group velocity of the phase-matched frequency components for the same conditions as in (a). Dots highlight the carrier frequencies for which the radiation group velocity is equal to that of the pump. 
stance, the circled segment in Fig. 1(a) shows the spectral support of the PBB generated in our experimental conditions, with each temporal spectral component dispersed with the same transverse wave vector.

In the general case the group velocity of the conical wave obtained with a coherent seeding at the carrier frequency $\omega_{0}$ can be extracted from the angular dispersion of the phase-matched radiation. Indeed $v_{g}\left(\omega_{0}\right)=1 /\left.\left(\mathrm{d} k_{z} / \mathrm{d} \omega\right)\right|_{\omega_{0}}$, where $k_{z}=\sqrt{|\mathbf{k}|^{2}-k_{\perp}^{2}}$ is the longitudinal phase-matched component of the amplified signal. In Fig. 1(b) the group velocity has been plotted versus $\omega$ for the same crystal tuning conditions considered in Fig. 1(a); the dots identify the carrier frequencies of the amplified wave packets for which the group velocity is equal to that of the pump pulse. In this work we have exploited the possibility of tuning the phase-matching curve so as to obtain a groupvelocity-matched interaction between the pump and the seed, thus maximizing the overall amplification gain. It is worth noting that in our specific operating conditions the group-velocity-matching constraint implies reshaping and amplification of the seed into a PBB (see Fig. 1).

We have experimentally realized an OPA by pumping a 4 -mm-long type I BBO crystal with the second harmonic pulse (405 nm, $100 \mathrm{fs}$ FWHM duration) from a Ti:sapphire laser source. The $100 \mathrm{fs}$ OPA seed obtained by frequency doubling the radiation generated by a commercial tunable noncollinear OPA (Topas, Light Conversion) was chosen to have a carrier wavelength at $600 \mathrm{~nm}\left(\sim 3.1 \times 10^{15} \mathrm{rad} \mathrm{Hz}\right)$. We identified the tuning angle condition [phase-matching described by the dashed curve in Fig. 1(a)] for which the interacting waves are group-velocity matched at their respective wavelengths [see Fig. 1(b)], i.e., $\theta_{t}=36.95^{\circ}$.

The first experiment demonstrates the reshaping into a PBB of a Gaussian seed. The Gaussian seed pulse was strongly focused collinearly with the pump onto the input facet of the nonlinear crystal by means of a long working distance, infinity corrected, $50 \times$, microscope objective (Plan APO 50× NIR, Mitutoyo), to obtain a Gaussian spatial intensity profile of $\sim 1.13 \mu \mathrm{m}$ width (half-width at $1 / e^{2}$ of maximum). Such a high spatial localization in the focus corresponds approximately to a spatial spectral width (at $1 / e^{2}$ ) of $1.84 \mu \mathrm{m}^{-1}$, thus guaranteeing an adequate distribution of the seed transverse $k$ vectors compatible with the phase-matched spatial frequencies of the OPA. Note that in the chosen configuration the angle between the collimated pump pulse direction and the seed wave vectors reached a value as wide as $11^{\circ}$. When the seed and pump pulses were spatially and temporally overlapped inside the nonlinear crystal, reshaping and amplification occurred. We recorded the near-field signal intensity profile at the output of the BBO with a modified Nikon D70 camera, by means of a long working distance, infinity corrected, $80 \times$ objective (Plan APO $80 \times$, Mitutoyo). The intensity distribution is shown in Fig. 2, while the seed input spatial intensity profile, measured at the input facet of the crystal, is presented in the inset for

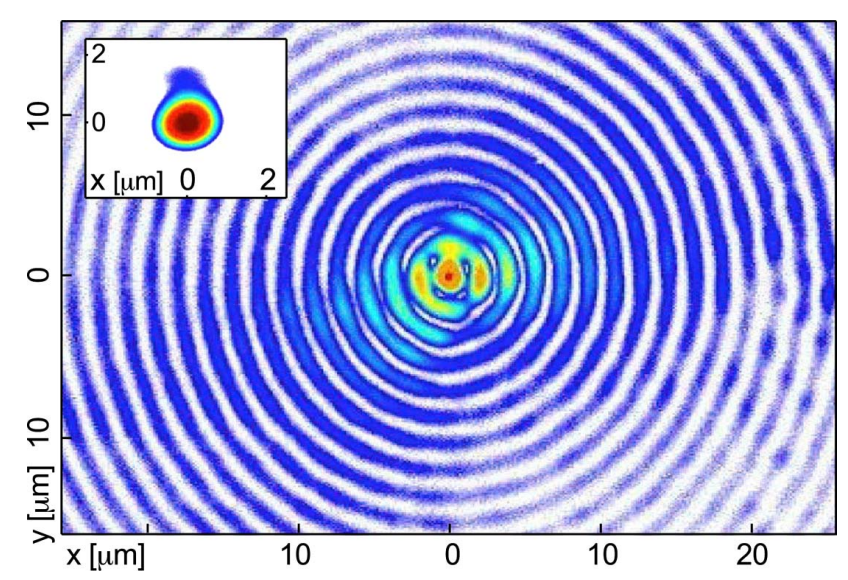

Fig. 2. (Color online) Near-field intensity distribution of the reshaped and amplified radiation in the case of strongly focused (Gaussian) seed pulse. Inset, near-field intensity profile of the input seed measured at the input facet of the crystal.

comparison. It is evident that the seed pulse has been reshaped into a conical wave, as confirmed by the clear Bessel-like pattern recorded at the crystal output. Starting from $0.7 \mathrm{~nJ}$ and $42 \mu \mathrm{J}$ energy for the seed and pump pulses, respectively, we measured $\sim 140 \mathrm{~nJ}$ of the amplified signal radiation, corresponding to a gain factor of $\sim 200$. Note that in Fig. 2 an asymmetry of the first Bessel circle is evident and is likely a consequence of phase distortions of the input seed pulse.

The spatiotemporal power spectrum of the amplified radiation was also recorded by means of a farfield diagnostic (see [17] for details of the technique) based on an imaging spectrometer (Shamrock, Andor) combined with a CCD camera (IDUS, Andor). The measured spectrum is indistinguishable from that obtained in the second experiment and shown in Fig. 3(b), to which we shall refer from here on. It is clear from that figure that the generated wave packet is a $\mathrm{PBB}$, as it results from the distribution of temporal frequencies, which all have the same transverse wave vector component, with a $10 \mathrm{~nm}$ bandwith centered around the seed carrier wavelength. This result perfectly matches the theoretical curve presented in Fig. 1(a) (circled segment).

In the second experiment the OPA was seeded with a $100 \mathrm{fs}, 600 \mathrm{~nm}$ carrier wavelength BXW. Such a conical wave has all temporal frequency components dispersed at the same angle $(\theta)$ and is thus characterized by an amplitude front perpendicular to the cone surface [16]. The seed was obtained by means of a $175^{\circ}$ base angle, fused silica axicon, inserted into a magnifying $4 f$ telescope system $\left(f_{1}=80 \mathrm{~cm}\right.$, $f_{2}=10 \mathrm{~cm}$ ). In this way the cone angle of the BXW generated by the axicon, namely, $\theta=1.15^{\circ}$, could be tuned (see [18] for details) and was here adjusted to the value of $\theta \sim 10.45^{\circ}$. Note that this corresponds to a central spatial frequency of $1.9 \mu \mathrm{m}^{-1}$, ensuring therefore the crossing of the seed spatiotemporal spectrum with the phase-matching curve selected in our experimental conditions [see inset in Fig. 3(a)].

The spatial reshaping process occurring here in the OPA is clearly evident from the comparison between 

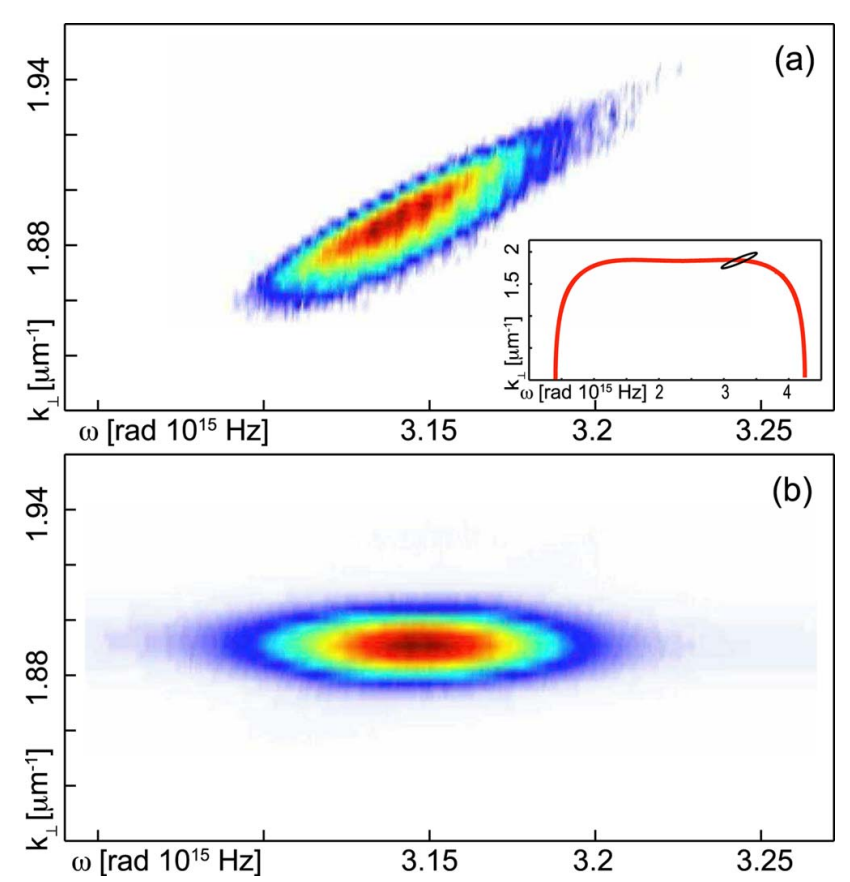

Fig. 3. (Color online) Spatiotemporal power spectra for (a) the BXW input seed and (b) the PBB reshaped and amplified pulses. Different colors correspond to different intensities of the power spectra, represented as a function of temporal and spatial frequency on the horizontal and vertical axis, respectively. In the inset, the seeding condition is sketched and compared with the phase-matching condition of the experiment.

Figs. 3(a) and 3(b). Figure 3(a) shows the power spectrum of the input seed. Considering the relation $k_{\perp}=\cos (\theta) n(\omega) \omega / c$ the spatiotemporal power spectrum of the seed BXW is clearly skewed. In contrast Fig. 3(b) shows the power spectrum of the amplified signal radiation, which has a single transverse wave vector component associated with all the temporal frequencies of the pulse. This clearly demonstrates the reshaping of the BXW seed into a PBB. In this case amplification occurs with a gain factor of $\sim 300$ (we measured an energy of $7.5 \mathrm{~nJ}$ for the input seed, and $2.2 \mu \mathrm{J}$ for the amplified signal pulse).

It is worth noting for completeness that during the parametric process where the seed is reshaped into a PBB and then amplified, the so-called "idler" conical wave is also generated. Such an idler has a carrier frequency dictated by the energy conservation law, namely, $\omega_{i}=\omega_{p}-\omega_{s}$, where $p, s$, and $i$ stand for pump, signal, and idler field, respectively, and is also a PBB being a frequency reversed copy of the signal (as also illustrated from the phase-matching curves of Fig. 1).

In conclusion, we have shown that a broadband collinear seeded OPA is able to efficiently reshape and amplify an input pulse signal into a PBB as a result of the spatiotemporal gain properties of the parametric amplification process. In particular, we have experimentally demonstrated in two different seeding configurations and by means of near-field and spa- tiotemporal spectral measurements, the reshaping of a Gaussian and BXW signal into an amplified PBB with a gain of 200 and 300, respectively. It is worth noting, finally, that PBBs are generally produced by means of linear methods, which may be affected by losses [19]. In contrast, here we have shown that PBBs can be efficiently generated in seeded OPAs, i.e., with gain.

The authors acknowledge support from the Consorzio Nazionale Interuniversitario per le Scienze fisiche della Materia (CNISM), project INNESCO, and from the Lithuanian State Science and Studies Foundation (ConteX project). M. Clerici acknowledges the support from Ministero dell'Istruzione, dell'Università e della Ricerca (MIUR), project RBIN04NYLH and thanks Lucia Caspani for fruitful discussions. P. Di Trapani acknowledges support from the Marie Curie Chair project STELLA MEXCCT-2005-025710.

\section{References}

1. H. Sõnajalg, M. Rätsep, and P. Saari, Opt. Lett. 22, 310 (1997).

2. Z. Liu and D. Fan, J. Mod. Opt. 45, 17 (1998).

3. D. McGloin and K. Dholakia, Contemp. Phys. 46, 15 (2005).

4. A. Averchi, D. Faccio, R. Berlasso, M. Kolesik, J. V. Moloney, A. Couairon, and P. Di Trapani, Phys. Rev. A 77, 021802 (2008).

5. P. Di Trapani, A. Beržanskis, S. Minardi, S. Sapone, and W. Chinaglia, Phys. Rev. Lett. 81, 5133 (1998).

6. M. Kolesik, E. Wright, and J. Moloney, Phys. Rev. Lett. 92, 253901 (2004).

7. P. D. Trapani, G. Valiulis, A. Piskarskas, O. Jedrkiewicz, J. Trull, C. Conti, and S. Trillo, Phys. Rev. Lett. 91, 093904 (2003).

8. D. Faccio, M. Porras, A. Dubietis, F. Bragheri, A. Couairon, and P. Di Trapani, Phys. Rev. Lett. 96, 193901 (2006).

9. P. Saari, and K. Reivelt, Phys. Rev. Lett. 79, 4135 (1997).

10. R. Boyd, Nonlinear Optics (Academic, 2002).

11. S. Longhi, Phys. Rev. E 69, 016606 (2004).

12. R. Butkus, S. Orlov, A. Piskarskas, V. Smilgevicius, and A. Stabinis, Opt. Commun. 244, 411 (2005).

13. G. Valiulis, A. Dubietis, and A. Piskarskas, Phys. Rev. A 77, 043824 (2008).

14. O. Jedrkiewicz, A. Picozzi, M. Clerici, D. Faccio, and P. D. Trapani, Phys. Rev. Lett. 97, 243903 (2006).

15. S. Orlov, A. Stabinis, V. Smilgevicius, G. Valiulis, and A. Piskarskas, Opt. Lett. 32, 68 (2007).

16. M. A. Porras, G. Valiulis, and P. D. Trapani, Phys. Rev. E 68, 016613 (2003).

17. D. Faccio, P. Di Trapani, S. Minardi, A. Bramati, F. Bragheri, C. Liberale, V. Degiorgio, A. Dubietis, and A. Matijosius, J. Opt. Soc. Am. B 22, 862 (2005).

18. V. Vaičaitis and S. Paulikas, Opt. Quantum Electron. 35, 1065 (2003).

19. J. Leach, G. M. Gibson, M. J. Padgett, E. E. G. McConnell, A. J. Wright, and J. M. Girkin, Opt. Express 14, 5581 (2006). 